\title{
Biochemistry and Cell Biology
}

\section{ATAD2 silencing decreases VEGFA secretion through targeting has-miR-520a to inhibit angiogenesis in colorectal cancer}

\begin{tabular}{|r|l|}
\hline Journal: & Biochemistry and Cell Biology \\
\hline Manuscript ID & bcb-2018-0081.R1 \\
\hline Date Submitted by the Author: & O6-Jun-2018 \\
\hline Complete List of Authors: & $\begin{array}{l}\text { Hong, Sen; The First Hospital of Jilin University } \\
\text { Chen, Si; The First Hospital of Jilin University } \\
\text { Wang, Xu; The First Hospital of Jilin University } \\
\text { Sun, Di; The First Hospital of Jilin University } \\
\text { Yan, Zhenkun; China-Japan Union Hospital of Jilin University } \\
\text { Tai, Jiandong; The First Hospital of Jilin University } \\
\text { Bi, Miaomiao; China-Japan Union Hospital of Jilin University, Department of } \\
\text { Ophthlmology }\end{array}$ \\
\hline Keyword: & ATAD2, colorectal cancer, angiogenesis, miR-520a-3p, VEGFA \\
\hline $\begin{array}{r}\text { Is the invited manuscript for } \\
\text { consideration in a Special } \\
\text { Issue? : }\end{array}$ & Not applicable (regular submission) \\
\hline
\end{tabular}


1 ATAD2 silencing decreases VEGFA secretion through targeting has-miR-520a to 2 inhibit angiogenesis in colorectal cancer

3 Sen Hong ${ }^{1}$, Si Chen ${ }^{1}, \mathrm{Xu}$ Wang $^{1}$, Di $\operatorname{Sun}^{1}$, Zhenkun Yan ${ }^{2}$, Jiandong Tai ${ }^{1, *}$, Miaomiao 4 $\mathrm{Bi}^{3, *}$

5 Author Affiliations: ${ }^{1}$ Department of Colorectal and Anal Surgery, The First Hospital 6 of Jilin University, Changchun 130021, People's Republic of China; ${ }^{2}$ Endoscopy 7 Center, China-Japan Union Hospital of Jilin University, Changchun 130033, People's 8 Republic of China; ${ }^{3}$ Department of Ophthlmology, China-Japan Union Hospital of 9 Jilin University, Changchun 130033, People's Republic of China.

"Corresponding authors: Dr. Miaomiao Bi, Department of Ophthlmology, China12 Japan Union Hospital of Jilin University, 126 Xiantai Avenue, Changchun 130033, 13 People's Republic of China, E-mail: miaomiao_b2008@sina.com, Tel: +86-43114 82951095, and Dr. Jiandong Tai, Department of Colorectal and Anal Surgery, The 15 First Hospital of Jilin University, 71 Xinmin Avenue, Changchun 130021, People's 16 Republic of China, E-mail: Jiandongtai@sina.com.

17

Running title: ATAD2 silencing inhibits angiogenesis in colorectal cancer 


\section{Abstract}

21 ATPase family AAA domain-containing protein 2 (ATAD2) is involved in various

22 types of cancers, including colorectal cancer. This study aimed to determine the role 23 of ATAD2 in angiogenesis in colorectal cancer. Here, we downregulated ATAD2 24 expression in HCT116 and SW480 cells, and collected the conditioned medium (CM) 25 from control and ATAD2-sienced cells. The effect of CM on human umbilical vein 26 endothelial cells (HUVEC) was evaluated by using CCK-8, wound healing, tube 27 formation, western blot, and dual-luciferase reporter assays. Our results showed that 28 the proliferation, migration and tube formation of HUVEC were reduced in presence 29 of ATAD2-sienced CM, and the levels of phosphorylated vascular endothelial growth 30 factor receptor 2 (P-VEGFR2), CD31, and CD34 were down-regulated. Mechanism 31 studies showed that ATAD2 silencing regulated the expression of vascular endothelial 32 growth factor A (VEGFA) and miR-520a. Moreover, we found that miR-520a could 33 bind to ATAD2, and its inhibitor partly reversed the alterations in HUVEC induced 34 by CM from ATAD2-sienced cells. In addition, we demonstrated that miR-520a 35 directly bound to $3^{\prime}$-UTR of VEGFA and inhibited its expression. Collectively, our 36 results indicate that ATAD2 inhibition suppresses VEGFA secretion by increasing 37 miR-520a levels. Our study suggests ATAD2 as a potential therapeutic target for 38 angiogenesis in colorectal cancer.

39 Key words: ATAD2; colorectal cancer; angiogenesis; miR-520a; VEGFA 
40

41

42

43

44

45

46

47

48

\section{Introduction}

Colorectal cancer is the third most common malignant tumor and the fourth leading cause of cancer death worldwide (Ferlay et al. 2015). The etiology of this caner is multifactorial and is thought to be associated with genetic and environmental factors (Kim et al. 2013). Currently, the main treatment of colorectal cancer consists of surgery, chemotherapy and radiation therapy, but the survival of colorectal cancer patients with advanced disease remains poor (Chen et al. 2014; Kou et al. 2015). Hence, it is necessary to develop new therapeutic strategies for the treatment of colorectal cancer.

ATAD2 (ATPase family, AAA domain containing 2), a member of ATPase family, contains an AAA domain that is important in regulating ATPase activity and protein multimerization, and a bromodomain that is capable of binding to acetylated histones (Krakstad et al. 2015). The structure of ATAD2 indicates that it has functions related to the regulation of cell proliferation, differentiation and apoptosis (Zheng et al. 2015). Previous studies demonstrated that ATAD2, as a cofactor for MYC, could promote tumor progression (Ciro et al. 2009). Overexpression of ATAD2 has been reported to be associated with poor prognosis in patients with cancer, such as breast cancer (Caron et al. 2010), lung cancer (Caron et al. 2010), gastric carcinoma (Zhang et al. 2016), ovarian cancer (Wan et al. 2014), and colorectal cancer (Hou et al. 2016). Moreover, our previous study demonstrated that knockdown of ATAD2 significantly inhibited migration, invasion, and epithelial-mesenchymal transition of colorectal cancer cells (Hong et al. 2016). However, the effect of ATAD2 on angiogenesis in colorectal cancer has not been yet characterized. 
63 In the present study, we silenced the expression of ATAD2 in HCT116 and SW480

64 cells and evaluated the effect of the conditioned medium (CM) from ATAD2-silenced

65 colorectal cancer cells on cell proliferation, migration, and tubule formation in human

66 umbilical vein endothelial cells (HUVEC). Furthermore, we demonstrated that

67 silencing of ATAD2 suppressed vascular endothelial growth factor A (VEGFA)

68 secretion by upregulating miR-520a in colorectal cancer.

\section{Materials and methods}

\section{Cells and cell culture}

71 HCT116, SW480, and HUVEC were purchased from Shanghai Zhong Qiao Xin Zhou

72 Biotechnology Co., Ltd. (Shanghai, China). All cells were cultured in Dulbecco's 73 modified Eagle's medium (DMEM; Gibco, Grand Island, NY, USA) supplemented 74 with $10 \%$ fetal bovine serum (FBS, Hyclone, Logan, UT, USA) and placed in an 75 incubator at $37^{\circ} \mathrm{C}$ and $5 \% \mathrm{CO}_{2}$.

\section{Vector construction and stable transfection}

77 The sequences of ATAD2 shRNA and non-target (negative control, NC) shRNA were 78 as follows: ATAD2 shRNA, 5'-GATCCCCGGCGATGGGTCATCAGTTATTCAAG 79 AGATAACTGATGACCCATCGCCTTTTT-3'; NC shRNA: 5'-GATCCCCTTC 80 TCCGAACGTGTCACGTTTCAAGAGAACGTGACACGTTCGGAGAATTTTT-3’.

81 They were cloned into the BamHI/HindIII site of pRNA H1.1 vector. Thereafter, the 82 recombinant plasmid was transfected into HCT116 and SW480 cells by 83 Lipofectamine 2000 (Invitrogen, Carlsbad, CA, USA). The drug resistant pooled cell 84 clones were obtained by G418 (Invitrogen) selection. The conditioned medium (CM) 
85 was collected from untreated cells, or cells stably transfected NC shRNA, or cells

86 stably transfected ATAD2 shRNA.

\section{Transient transfection and VEGFA treatment}

88 MiR-520a mimics and inhibitor were synthesized by GenePharma (Shanghai, China) 89 and transfected into HCT116 and SW480 cells by Lipofectamine 2000 (Invitrogen). 90 VEGFA was obtained from Sino Biological Inc. (Beijing, China) and dissolved in 91 sterile water. $20 \mathrm{ng} / \mathrm{ml}$ VEGFA was mixed with $\mathrm{CM}$ from HCT116 with ATAD2 92 knockdown to culture HUVEC.

101

\section{Real-time PCR}

Total RNA was isolated from cells using TriPure reagent (Bioteke, Beijing, China) and converted to cDNA by reverse transcription-PCR. Mir-520a RT primer: GTTGGCTCTGGTGCAGGGTCCGAGGTATTCGCACCAGAGCCAACACAGTC.

7 Then the cDNA was amplified by real-time PCR. The primer sequences were as follows: ATAD2 forward, GTGGGAAATAGTTGGACCGACA, reverse, CGGAATGGGGTTGTCAGAAG; mir-520a forward: GTACGAAAGTGCTTCCCTTTGG， reverse， GTGCAGGGTCCGAGGTATTC; CD31 forward: AAGATAGCCTCAAAGTCGGA, reverse, TCAAACTGGGCATCATAAGA; CD34 forward: GTCTTCCACTCGGTGCGTCTC, reverse, GTTCCCTGGGTAGGTAACTCT; $\quad \beta$-actin forward, CTTAGTTGCGTTACACCCTTTCTTG, reverse, CTGTCACCTTCACCGTTCCAGTTT. The reaction was carried out using an Exicycler 96 Bioneer (Bioneer Corporation, Daejoen, Korea). The relative expression of gene was calculated by the $2^{-\Delta \Delta \mathrm{Ct}}$ method. 
108

109

110

111

112

113

114

115

116

117

118

119

120

121

122 (Beijing Liuyi Biotechnology Co., Ltd., Beijing, China). The intensity of the bands

123 was evaluated by densitometric analysis and each band was normalized to $\beta$-actin

124 signal.

\section{Enzyme-linked immunosorbent assay (ELISA)}

126 The content of VEGFA was detected using a commercial ELISA kit (MultiSciences

127 Biotech Co., Ltd., Hangzhou, China) according to the manufacturer's instructions.

\section{Cell counting kit (CCK)-8 assay}

129 Cell proliferation was determined by a CCK-8 kit (Beyotime Institute of 130 Biotechnology). In brief, cells $\left(3 \times 10^{3}\right.$ per well) were seeded into 96 -well plates in 
131 quintuplicate. After treatment for 24, 48, 72, and $96 \mathrm{~h}$. The supernatant was discarded

132 at the indicated time point. Then $100 \mu \mathrm{l}$ of complete medium and $10 \mu \mathrm{l}$ of CCK-8

133 solution added to each well for $1 \mathrm{~h}$ at $37^{\circ} \mathrm{C}$. The optical density was detected at 450

$134 \mathrm{~nm}$ with a microplate reader (Biotek, Winooski, VT, USA).

\section{Scratch wound healing assay}

136 Cell migration was evaluated by scratch wound healing assay. Briefly, cells were

137 stimulated with $1 \mu \mathrm{g} / \mathrm{ml}$ mitomycin C (Sigma Aldrich, St Louis, USA) for $1 \mathrm{~h}$ when

138 they reached full confluence. After treatment for $24 \mathrm{~h}$. Scratch wounds were made

139 with sterile $200 \mu$ pipette tips. Cells were then cultured in serum-free medium and

140 photographed with an inverted phase-contrast microscope (Olympus, Tokyo, Japan) at

1410 and $24 \mathrm{~h}$. The cell migration rate was calculated based on the distance between the

142 two fronts of the wound.

\section{Tube formation assay}

144 Angiogenesis was assessed by tube formation assay. Briefly, cells $\left(3 \times 10^{4}\right.$ per well $)$

145 were dispensed into 96-well plates coated with Matrigel (BD Bioscience, Franklin

146 Lakes, NJ, USA). After treatment for $18 \mathrm{~h}$. The image was captured using a

147 microscope (Olympus) and the number of tube formation was counted.

148 Dual-luciferase reporter assay

$149293 \mathrm{~T}$ cells were co-transfected with pmirGLO vector including the 3' UTR fragment

150 or 3' untranslated region (UTR) mutant fragment of ATAD2, and miR-520 mimics or

151 miR-520 NC mimics for $48 \mathrm{~h}$. Renilla and firefly luciferase activities were assayed 
152 using the Dual Luciferase Reporter Assay System (Promega, Madison, WI, USA).

153 The ratios of firefly and renilla luciferase activity were calculated.

\section{Statistical analysis}

155 Each experiment was repeated three times and the results were presented as mean \pm

156 standard deviation (SD). Statistical differences were analyzed using one or two-way

157 ANOVA by GraphPad prism 7 software (GraphPad Software, La Jolla, CA, USA).

158 Values of P less than 0.05 were considered statistically significant.

159

160 Results

161 Silencing of ATAD2 in colorectal cancer cells

162 We stably knocked down the expression of ATAD2 in HCT116 and SW480 cells. As

163 shown in Figure 1A and B, results of western blot and real-time PCR assays showed

164 that ATAD2 expression was significantly decreased in ATAD2-silenced colorectal

165 cancer cells.

166 Proliferation and migration of HUVEC were attenuated after being cultured in

167 CM from ATAD2-silenced colorectal cancer cells

168 We collected CM from HCT116 or SW480 cells with or without ATAD2 knockdown.

169 As shown in Figure 2A, results from CCK-8 assay revealed that the proliferation

170 ability of HUVEC was inhibited when cultured in CM from ATAD2-silenced

171 colorectal cancer cells. Moreover, the migration ability of HUVEC was suppressed

172 when cultured in CM from ATAD2-silenced HCT116 or SW480 cells (Figure 2B). 
173 Tube formation of HUVEC was inhibited after being cultured in CM from 174 ATAD2-silenced colorectal cancer cells

175 Furthermore, we evaluated the influence of CM on the tube formation of HUVEC. As 176 shown in Figure 3A, the number of tube formation in HUVEC cultured in ATAD2177 silenced CM was reduced. In addition, western bolt analysis revealed a decreased 178 expression of P-VEGFR2, CD31, and CD34 in HUVEC exposed to ATAD2-silenced $179 \mathrm{CM}$ (Figure 3B). The results from real-time PCR indicated that the alterations in the 180 mRNA levels of CD31 and CD34 were in accordance with their protein levels (Figure $1813 \mathrm{C})$.

182 183

Knockdown of ATAD2 suppressed the level of VEGFA through targeting miR$520 a$

We subsequently studied the underlying molecular mechanisms of ATAD2 in colorectal cancer cells. As shown in Figure 4A, we found that the level of VEGFA in the supernatants of ATAD2-silenced CT116 and SW480 cells was decreased. Realtime PCR analysis showed an increase in miR-520a in colorectal cancer cells after knockdown of ATAD2 (Figure 4B). Similarly, VEGFA expression in ATAD2silenced colorectal cancer cells was inhibited following ATAD2 silencing (Figure 4C). Moreover, the results of western blot showed that miR-520a mimics could inhibit the expression of VEGFA, and miR-520a inhibitor could promote VEGFA expression in HCT116 and SW480 cells (Figure 4D). Furthermore, we demonstrated that miR-520a directly targeted the 3'-UTR of ATAD2 in 293 T cells by dual-Luciferase reporter assay (Figure 4E). Moreover, we found that miR-520a inhibitor could reverse the inhibition of VEGFA level and cell migration and tube formation caused by CM from ATAD2-sienced HCT116 cells (Figure 5A-C). 
197 Administration of VEDFA relieved the inhibiting effect of CM on HUVEC

198 Lastly, we demonstrated that miR-520a directly targeted the 3'-UTR of VEGFA in $199293 \mathrm{~T}$ cells (Figure 6A). Moreover, as shown in Figure 6B-D, administration of 200 VEDFA reversed the inhibition of proliferation, migration, and tube formation caused 201 by CM from ATAD2-silenced HCT116 cells in HUVEC.

\section{Discussion}

204 Metastasis is a main cause of mortality in patients with colorectal cancer (Cierniewski 205 et al. 2010), and angiogenesis plays a critical role in this process (Harris et al. 2012).

206 Numerous studies have demonstrated that ATAD2 was involved in cell growth in 207 several cancers, such as cervical cancer (Zheng et al. 2015), hepatocellular carcinoma 208 (Lu et al. 2015), breast cancer (Kalashnikova et al. 2010), and colorectal cancer 209 (Hong et al. 2016). However, the role of ATAD2 in angiogenesis during tumor 210 development has not been reported yet. In the present study, we demonstrated that the 211 proliferation, migration, and tube formation in HUVEC cultured in CM from 212 ATAD2-silenced colorectal cancer cells were significantly inhibited, which was 213 accompanied with downregulation of vascular endothelial cell markers, including P214 VEGFR2, CD31, and CD34, indicating that ATAD2 plays a role in the angiogenesis 215 in colorectal cancer.

216 Multiple signaling pathways are implicated in the regulation of angiogenesis, such as 217 VEGF (Hicklin and Ellis 2005), Delta-like 4 (Dll4)/Notch (Liu et al. 2014), Wnt 218 (Choi et al. 2012), and dimethylarginine dimethylaminohydrolase/asymmetric 219 dimethylarginine (DDAH/ADMA) (Fiedler and Wojciak-Stothard 2009) pathways. 
220 Among angiogenic factors, VEGF family is the most important one involved in the 221 angiogenesis, which includes VEGFA, VEGFB, VEGFC, VEGFD and a structurally 222 related molecule, placental growth factor (PlGF) (Murukesh et al. 2010; Varinska et al. 223 2015). The most intensively studied member of VEGF family is VEGFA, which can 224 bind to both VEGF receptor 1 (VEGFR-1) and -2 (VEGFR-2) to promote 225 angiogenesis (Shibuya 2013). VEGFA is up-regulated in a variety of cancers, 226 including colorectal cancer, and it is used as an indicator for poor prognosis (Martins 227 et al. 2013). Moreover, numerous VEGFA inhibitors have been reported in the 228 treatment of cancers through inhibition of tumor angiogenesis, such as bevacizumab 229 and ranibizumab (Ferrara et al. 2007). In the present study, we found that knockdown 230 of ATAD2 decreased the level of VEGFA in colorectal cancer cells. Moreover, the 231 effect of CM from ATAD2-silenced colorectal cancer cells on HUVEC could be 232 reversed by adding exogenous VEGFA.

233 MicroRNAs are a class of non-coding RNAs of about 22 nucleotides in length, and 234 they function as posttranscriptional gene regulators (Diederichs and Haber 2007). 235 Previous studies have shown that miRNAs, such as such as miR-15a, miR-16, and 236 miR-101, contribute to angiogenesis by targeting VEGF (Sun et al. 2013; Zhang et al. 237 2013). Recent studies show that miR-520a can suppress cell proliferation in non-small 238 cell lung cancer (Yu et al. 2015) and hepatocellular carcinoma (Dong et al. 2015), 239 suggesting it as a tumor suppressor. In this study, we found miR-520a as a miRNA 240 regulated by ATAD2. Our data showed that ATAD2 inhibition lead to miR-520a 241 upregulation. Similar phenomenon was observed in hepatocellular carcinoma by 242 Huang and co-workers (Huang et al. 2016). Our study together with a study from Ye 243 et al. (Ye et al. 2015) demonstrated VEGFA as a direct target of miR-520a. Although 244 how ATAD2 regulates miR-520a is unclear, by analyzing the sequence of ATAD2 
245 (TargetScan, Version 7.2), we find that ATAD2 possesses sequence for miR-520a 246 recognition. Our additional dual-Luciferase reporter assay shows that ATAD2 can 247 bind to miR-520a. It is possible that the mRNA of ATAD2 gene may act as a 248 competitive RNA to sponge miR-520a from targeting VEGFA. Further experiments 249 will be performed in the near future to validate this hypothesis.

250 In summary, our data demonstrate that the proliferation, migration, and tube 251 formation of HUVEC was suppressed after being cultured in CM from ATAD2252 silenced colorectal cancer cells. Moreover, knockdown of ATAD2 downregulates the 253 level of VEGFA by upregulating miR-520a. Our results suggest ATAD2 as a 254 potential therapeutic target to inhibit angiogenesis in colorectal cancer.

255

256 Conflict of interest

257 All authors declare that they have no conflicts of interest.

\section{Acknowledgements}

259 The authors are grateful to staff from the High-tech Zone Laboratory of Public Test 260 and Analysis Service (Shenyang, China) for their assistance in the experiments and 261 English editing. 
262

263 264 265 266 267 268 269

270 271

272

273

274

275

276

277

278

279

280

281

282

283

284 285 286

\section{References}

Caron, C., Lestrat, C., Marsal, S., Escoffier, E., Curtet, S., Virolle, V., Barbry, P., Debernardi, A., Brambilla, C., Brambilla, E., Rousseaux, S., and Khochbin, S. 2010. Functional characterization of ATAD2 as a new cancer/testis factor and a predictor of poor prognosis in breast and lung cancers. Oncogene 29(37): 51715181. doi: 10.1038/onc.2010.259.

Chen, C., Wang, L., Liao, Q., Xu, L., Huang, Y., Zhang, C., Ye, H., Xu, X., Ye, M., and Duan, S. 2014. Association between six genetic polymorphisms and colorectal cancer: a meta-analysis. Genet Test Mol Biomarkers 18(3): 187-195. doi: $10.1089 /$ gtmb.2013.0425.

Choi, H.J., Park, H., Lee, H.W., and Kwon, Y.G. 2012. The Wnt pathway and the roles for its antagonists, DKKS, in angiogenesis. IUBMB Life 64(9): 724-731. doi: 10.1002/iub.1062.

Cierniewski, C.S., Papiewska-Pajak, I., Malinowski, M., Sacewicz-Hofman, I., Wiktorska, M., Kryczka, J., Wysocki, T., Niewiarowska, J., and Bednarek, R. 2010. Thymosin beta4 regulates migration of colon cancer cells by a pathway involving interaction with Ku80. Ann N Y Acad Sci 1194: 60-71. doi: 10.1111/j.1749$6632.2010 .05480 . x$

Ciro, M., Prosperini, E., Quarto, M., Grazini, U., Walfridsson, J., McBlane, F., Nucifero, P., Pacchiana, G., Capra, M., Christensen, J., and Helin, K. 2009. ATAD2 is a novel cofactor for MYC, overexpressed and amplified in aggressive tumors. Cancer Res 69(21): 8491-8498. doi: 10.1158/0008-5472.CAN-09-2131.

Diederichs, S., and Haber, D.A. 2007. Dual role for argonautes in microRNA processing and posttranscriptional regulation of microRNA expression. Cell 131(6): 1097-1108. doi: 10.1016/j.cell.2007.10.032. 
287 Dong, Y., Zou, J., Su, S., Huang, H., Deng, Y., Wang, B., and Li, W. 2015. 288 MicroRNA-218 and microRNA-520a inhibit cell proliferation by downregulating 289 E2F2 in hepatocellular carcinoma. Mol Med Rep 12(1): 1016-1022. doi: $290 \quad 10.3892 / \mathrm{mmr} .2015 .3516$.

291 Ferlay, J., Soerjomataram, I., Dikshit, R., Eser, S., Mathers, C., Rebelo, M., Parkin, 292 D.M., Forman, D., and Bray, F. 2015. Cancer incidence and mortality worldwide: 293 sources, methods and major patterns in GLOBOCAN 2012. Int J Cancer 136(5): 294 E359-386. doi: 10.1002/ijc.29210.

295 Ferrara, N., Mass, R.D., Campa, C., and Kim, R. 2007. Targeting VEGF-A to treat 296 cancer and age-related macular degeneration. Annu Rev Med 58: 491-504. doi: $297 \quad$ 10.1146/annurev.med.58.061705.145635.

298 Fiedler, L.R., and Wojciak-Stothard, B. 2009. The DDAH/ADMA pathway in the 299 control of endothelial cell migration and angiogenesis. Biochem Soc Trans 37(Pt 300 6): 1243-1247. doi: 10.1042/BST0371243.

301 Harris, L.G., Pannell, L.K., Singh, S., Samant, R.S., and Shevde, L.A. 2012. Increased 302 vascularity and spontaneous metastasis of breast cancer by hedgehog signaling 303 mediated upregulation of cyr61. Oncogene 31(28): 3370-3380. doi: $304 \quad 10.1038 /$ onc.2011.496.

305 Hicklin, D.J., and Ellis, L.M. 2005. Role of the vascular endothelial growth factor 306 pathway in tumor growth and angiogenesis. J Clin Oncol 23(5): 1011-1027. doi: $307 \quad 10.1200 / \mathrm{JCO} .2005 .06 .081$.

308 Hong, S., Bi, M., Yan, Z., Sun, D., Ling, L., and Zhao, C. 2016. Silencing of ATPase 309 family AAA domain-containing protein 2 inhibits migration and invasion of 310 colorectal cancer cells. Neoplasma 63(6): 846-855. doi: 10.4149/neo_2016_603. 
311 Hou, M., Huang, R., Song, Y., Feng, D., Jiang, Y., and Liu, M. 2016. ATAD2 312 overexpression is associated with progression and prognosis in colorectal cancer. $313 \quad$ Jpn J Clin Oncol 46(3): 222-227. doi: 10.1093/jjco/hyv195.

314 Huang, J., Yang, J., Lei, Y., Gao, H., Wei, T., Luo, L., Zhang, F., Chen, H., Zeng, Q., 315 and Guo, L. 2016. An ANCCA/PRO2000-miR-520a-E2F2 regulatory loop as a 316 driving force for the development of hepatocellular carcinoma. Oncogenesis 5(5): 317 e229. doi: 10.1038/oncsis.2016.22.

318 Kalashnikova, E.V., Revenko, A.S., Gemo, A.T., Andrews, N.P., Tepper, C.G., Zou, 319 J.X., Cardiff, R.D., Borowsky, A.D., and Chen, H.W. 2010. ANCCA/ATAD2 320 overexpression identifies breast cancer patients with poor prognosis, acting to drive 321 proliferation and survival of triple-negative cells through control of B-Myb and 322 EZH2. Cancer Res 70(22): 9402-9412. doi: 10.1158/0008-5472.CAN-10-1199.

323 Kim, E., Coelho, D., and Blachier, F. 2013. Review of the association between meat 324 consumption and risk of colorectal cancer. Nutr Res 33(12): 983-994. doi: $325 \quad$ 10.1016/j.nutres.2013.07.018.

326 Kou, C., Zhou, T., Han, X., Zhuang, H., and Qian, H. 2015. LRIG1, a 3p tumor 327 suppressor, represses EGFR signaling and is a novel epigenetic silenced gene in 328 colorectal cancer. Biochem Biophys Res Commun 464(2): 519-525. doi: $329 \quad 10.1016 /$ j.bbrc.2015.06.173.

330 Krakstad, C., Tangen, I.L., Hoivik, E.A., Halle, M.K., Berg, A., Werner, H.M., 331 Raeder, M.B., Kusonmano, K., Zou, J.X., Oyan, A.M., Stefansson, I., Trovik, J., 332 Kalland, K.H., Chen, H.W., and Salvesen, H.B. 2015. ATAD2 overexpression 333 links to enrichment of B-MYB-translational signatures and development of 334 aggressive endometrial carcinoma. Oncotarget 6(29): 28440-28452. doi: 33510.18632 oncotarget.4955. 
336 Liu, Z., Fan, F., Wang, A., Zheng, S., and Lu, Y. 2014. Dll4-Notch signaling in 337 regulation of tumor angiogenesis. J Cancer Res Clin Oncol 140(4): 525-536. doi: 10.1007/s00432-013-1534-x.

339 Lu, W.J., Chua, M.S., and So, S.K. 2015. Suppression of ATAD2 inhibits 340 hepatocellular carcinoma progression through activation of p53- and p38-mediated 341 apoptotic signaling. Oncotarget 6(39): 41722-41735. doi: $342 \quad 10.18632 /$ oncotarget.6152.

343 Martins, S.F., Garcia, E.A., Luz, M.A., Pardal, F., Rodrigues, M., and Filho, A.L. 344 2013. Clinicopathological correlation and prognostic significance of VEGF-A, 345 VEGF-C, VEGFR-2 and VEGFR-3 expression in colorectal cancer. Cancer 346 Genomics Proteomics 10(2): 55-67.

347 Murukesh, N., Dive, C., and Jayson, G.C. 2010. Biomarkers of angiogenesis and their 348 role in the development of VEGF inhibitors. Br J Cancer 102(1): 8-18. doi: $349 \quad 10.1038 /$ sj.bjc.6605483.

350 Shibuya, M. 2013. VEGFR and type-V RTK activation and signaling. Cold Spring 351 Harb Perspect Biol 5(10): a009092. doi: 10.1101/cshperspect.a009092.

352 Sun, C.Y., She, X.M., Qin, Y., Chu, Z.B., Chen, L., Ai, L.S., Zhang, L., and Hu, Y. 353 2013. miR-15a and miR-16 affect the angiogenesis of multiple myeloma by 354 targeting VEGF. Carcinogenesis 34(2): 426-435. doi: 10.1093/carcin/bgs333.

355 Varinska, L., Gal, P., Mojzisova, G., Mirossay, L., and Mojzis, J. 2015. Soy and 356 breast cancer: focus on angiogenesis. Int J Mol Sci 16(5): 11728-11749. doi: $357 \quad 10.3390 /$ ijms 160511728.

358 Wan, W.N., Zhang, Y.X., Wang, X.M., Liu, Y.J., Zhang, Y.Q., Que, Y.H., and Zhao, 359 W.J. 2014. ATAD2 is highly expressed in ovarian carcinomas and indicates poor 360 prognosis. Asian Pac J Cancer Prev 15(6): 2777-2783. 
361 Ye, X., Fan, F., Bhattacharya, R., Bellister, S., Boulbes, D.R., Wang, R., Xia, L., Ivan, 362 C., Zheng, X., Calin, G.A., Wang, J., Lu, X., and Ellis, L.M. 2015. VEGFR-1 363 Pseudogene Expression and Regulatory Function in Human Colorectal Cancer 364 Cells. Mol Cancer Res 13(9): 1274-1282. doi: 10.1158/1541-7786.MCR-15-0061. 365 Yu, J., Tan, Q., Deng, B., Fang, C., Qi, D., and Wang, R. 2015. The microRNA-520a$3663 p$ inhibits proliferation, apoptosis and metastasis by targeting MAP3K2 in non367 small cell lung cancer. Am J Cancer Res 5(2): 802-811.

368 Zhang, J., Han, C., Zhu, H., Song, K., and Wu, T. 2013. miR-101 inhibits 369 cholangiocarcinoma angiogenesis through targeting vascular endothelial growth 370 factor (VEGF). Am J Pathol 182(5): 1629-1639. doi: 10.1016/j.ajpath.2013.01.045. 371 Zhang, M., Zhang, C., Du, W., Yang, X., and Chen, Z. 2016. ATAD2 is 372 overexpressed in gastric cancer and serves as an independent poor prognostic 373 biomarker. Clin Transl Oncol 18(8): 776-781. doi: 10.1007/s12094-015-1430-8.

374 Zheng, L., Li, T., Zhang, Y., Guo, Y., Yao, J., Dou, L., and Guo, K. 2015. Oncogene 375 ATAD2 promotes cell proliferation, invasion and migration in cervical cancer. 376 Oncol Rep 33(5): 2337-2344. doi: 10.3892/or.2015.3867. 
379

380

381

382

383

384

385

386

387

388

389

390

391

392

393

394

395

396

397

398

39

400

401

Figure legends

Figure 1. Knockdown of ATAD2 in colorectal cancer cells. The expression of ATAD2 was evaluated in HCT116 and SW480 cells following silencing of ATAD2 by western blotting (A) and real-time PCR (B). Data were present as mean $\pm \mathrm{SD}$. $* * * \mathrm{P}<0.001$

\section{Figure 2. HUVEC proliferation and migration were inhibited after culture in}

CM from ATAD2-silenced colorectal cancer cells. Proliferation and migration ability of HUVEC cultured in CM was determined by CCK-8 assay (A) and wound healing assay (B), respectively. H-CM, HCT116 conditioned medium; H-NC, HCT116 negative control conditioned medium; H-ATAD2-shRNA, HCT116 ATAD2-shRNA conditioned medium. S-CM, SW480 conditioned medium; S-NC, SW480 negative control conditioned medium; S-ATAD2-shRNA, SW480 ATAD2shRNA conditioned medium. Data were present as mean $\pm \mathrm{SD} .{ }^{*} \mathrm{P}<0.05 ; * * \mathrm{P}<0.01$; $* * * \mathrm{P}<0.001$.

Figure 3. The angiogenesis of HUVEC was suppressed after culture in CM from ATAD2-silenced colorectal cancer cells. The capillary tube formation of HUVEC cultured in CM was evaluated by tube formation assay (A). Expression levels of PVEGFR2, VEGFR2, CD31, and CD34 were detected by western blotting (B). The mRNA expression levels of CD31 and CD34 were determined through real-time PCR (C). H-SFM, HCT116 serum free medium; H-CM, HCT116 conditioned medium; HNC, HCT116 negative control conditioned medium; H-ATAD2-shRNA, HCT116 ATAD2-shRNA conditioned medium. S-SFM, SW480 serum free medium; S-CM, SW480 conditioned medium; S-NC, SW480 negative control conditioned medium; S- 
402 ATAD2-shRNA, SW480 ATAD2-shRNA conditioned medium. Data were present as 403 mean $\pm \mathrm{SD} . * \mathrm{P}<0.05 ; * * \mathrm{P}<0.01 ; * * * \mathrm{P}<0.001$

404 405 406 407 408 409 410

Figure 4. ATAD2 regulated the level of VEGFA via miR-520a in colorectal cancer cells. Levels of VEGFA in the supernatant of ATAD2-silenced HCT116 or SW480 cells were determined by ELISA kit (A). Real-time PCR was employed to detect miR-520a expression in colorectal cancer cells with or without ATAD2 knockdown (B). The expression of VEGFA in HCT116 or SW480 cells following silencing of ATAD2 was assessed through western blotting (C). The effect of miR520a mimics or inhibitor on the expression of VEGFA in HCT116 or SW480 cells was evaluated by western blotting (D). The effect of miR-520a mimics on luciferase activity of ATAD2 3'-UTR was determined (E). Data were present as mean $\pm \mathrm{SD}$. $* \mathrm{P}<0.05 ; * * \mathrm{P}<0.01 ; * * * \mathrm{P}<0.001$

Figure 5. MiR-520a inhibitor reversed the inhibiting effect of CM from ATAD2silenced HCT116 cells on HUVEC. Levels of VEGFA in the supernatant of ATAD2-silenced HCT116 were determined by ELISA kit (A). Migration and capillary tube formation of HUVEC were assessed by wound healing (B) and tube formation assays (C), respectively. H-NC, HCT116 negative control conditioned medium; H-ATAD2-shRNA, HCT116 ATAD2-shRNA conditioned medium. HATAD2-shRNA + NC inhibitor, HCT116 ATAD2-shRNA conditioned medium + . negative control inhibitor. H-ATAD2-shRNA + mir-520a inhibitor, HCT116 ATAD2-shRNA conditioned medium + mir-520a inhibitor. Data were present as mean $\pm \mathrm{SD} .{ }^{*} \mathrm{P}<0.05 ; * * \mathrm{P}<0.01 ; * * * \mathrm{P}<0.001$

Figure 6. Treatment with VEGFA reversed the inhibiting effect of $\mathrm{CM}$ from ATAD2-silenced HCT116 cells on HUVEC. The effect of miR-520a mimics on 
426 luciferase activity of VEGFA 3'-UTR was assessed (A). Proliferation, migration, and 427 capillary tube formation of HUVEC was determined by CCK-8 (B), wound healing 428 (C), and tube formation assays (D), respectively. H-SFM, HCT116 serum free 429 medium; H-NC, HCT116 negative control conditioned medium; H-ATAD2-shRNA, 430 HCT116 ATAD2-shRNA conditioned medium. H-ATAD2-shRNA + VEGFA, 431 HCT116 ATAD2-shRNA conditioned medium $+20 \mathrm{ng} / \mathrm{ml}$ VEGFA. Data were 432 present as mean $\pm \mathrm{SD} . * * \mathrm{P}<0.01 ; * * * \mathrm{P}<0.001$ 
A
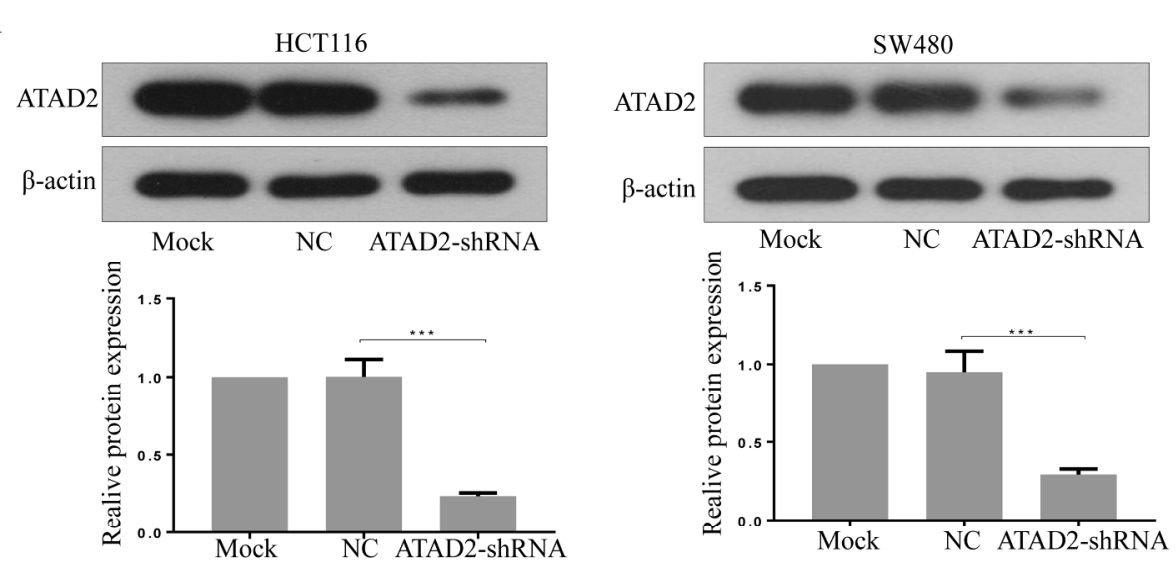

$\mathrm{B}$
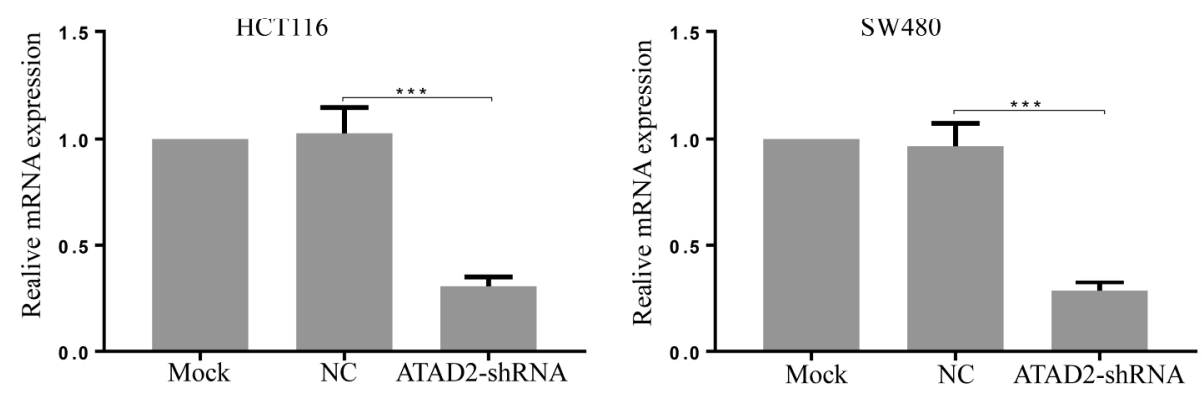

Figure 1. Knockdown of ATAD2 in colorectal cancer cells. The expression of ATAD2 was evaluated in HCT116 and SW480 cells following silencing of ATAD2 by western blotting (A) and real-time PCR (B). Data were present as mean $\pm \mathrm{SD}$. $* * * \mathrm{P}<0.001$.

$205 \times 166 \mathrm{~mm}(300 \times 300 \mathrm{DPI})$ 
A
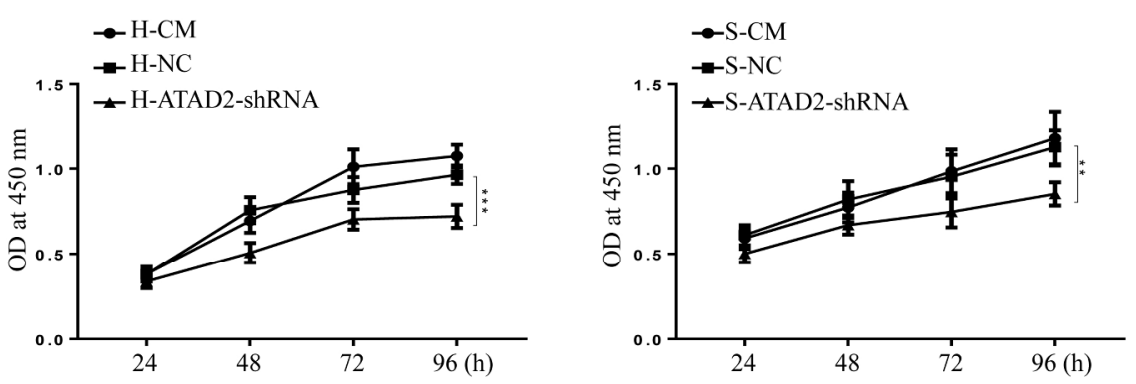

B
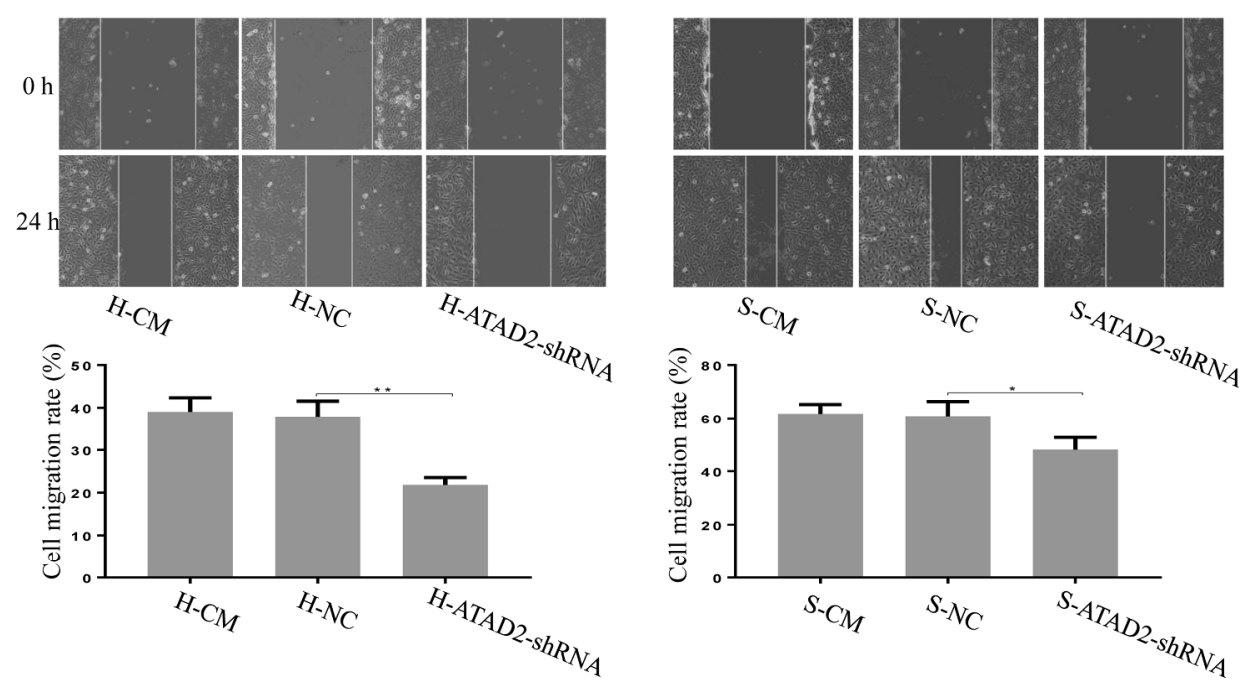

Figure 2. HUVEC proliferation and migration were inhibited after culture in CM from ATAD2-silenced colorectal cancer cells. Proliferation and migration ability of HUVEC cultured in CM was determined by CCK- 8 assay $(A)$ and wound healing assay (B), respectively. H-CM, HCT116 conditioned medium; H-NC, HCT116 negative control conditioned medium; H-ATAD2-shRNA, HCT116 ATAD2-shRNA conditioned medium. S-CM, SW480 conditioned medium; S-NC, SW480 negative control conditioned medium; S-ATAD2-shRNA, SW480 ATAD2-shRNA conditioned medium. Data were present as mean \pm SD. $* P<0.05 ; * * P<0.01 ; * * * P<0.001$.

$217 \times 186 \mathrm{~mm}(300 \times 300 \mathrm{DPI})$ 
A
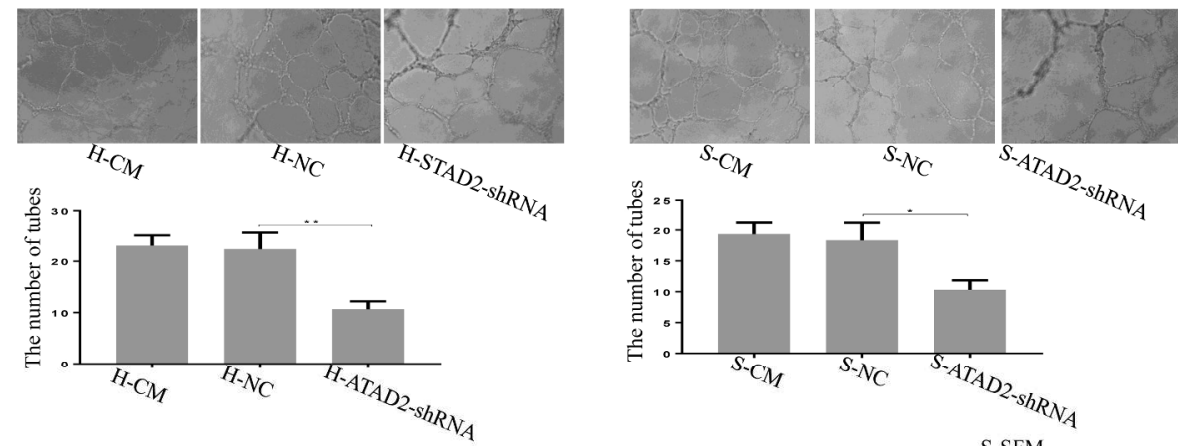

B
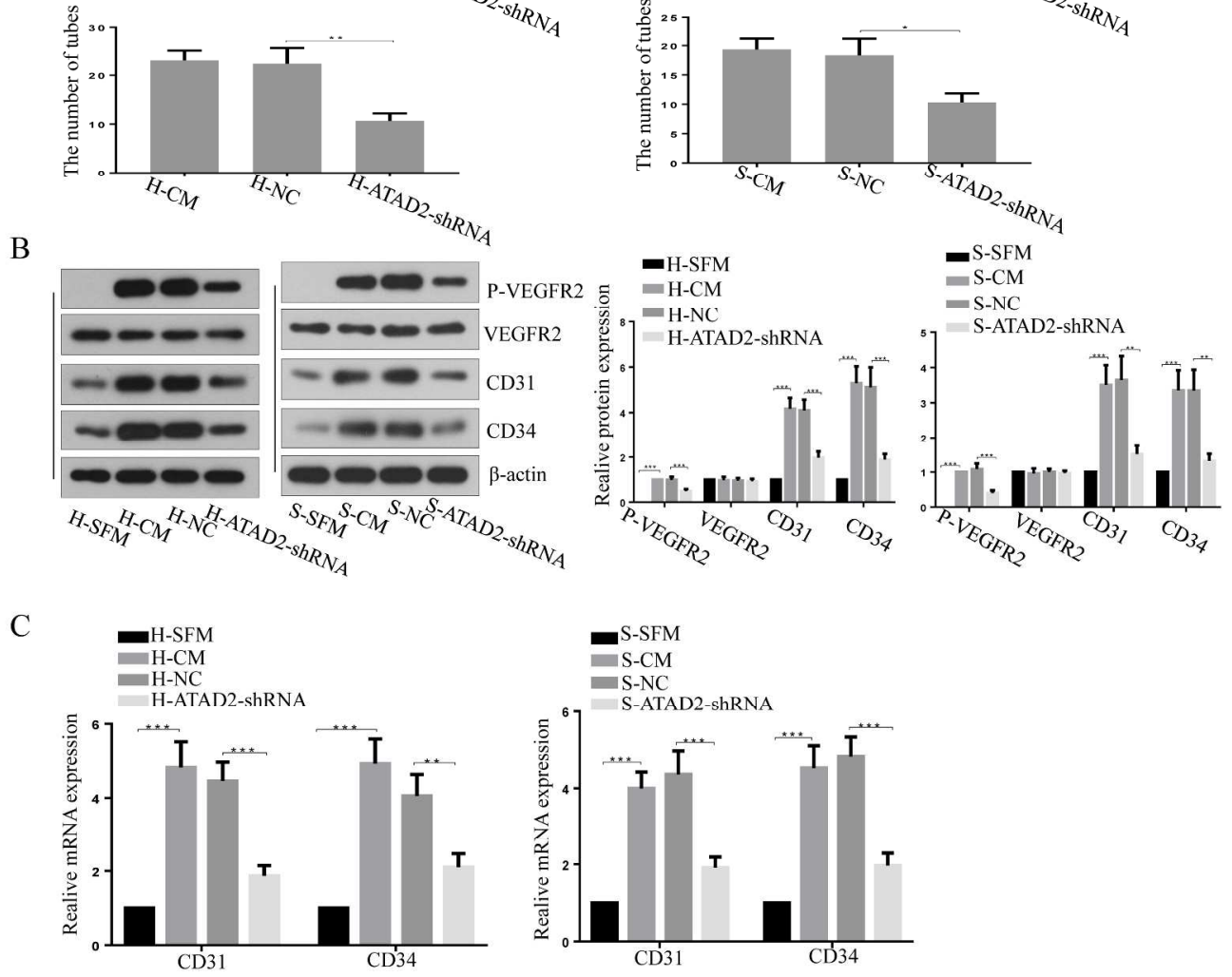

Figure 3. The angiogenesis of HUVEC was suppressed after culture in CM from ATAD2-silenced colorectal cancer cells. The capillary tube formation of HUVEC cultured in CM was evaluated by tube formation assay (A). Expression levels of P-VEGFR2, VEGFR2, CD31, and CD34 were detected by western blotting (B). The mRNA expression levels of CD31 and CD34 were determined through real-time PCR (C). H-SFM, HCT116 serum free medium; H-CM, HCT116 conditioned medium; H-NC, HCT116 negative control conditioned medium; H-ATAD2-shRNA, HCT116 ATAD2-shRNA conditioned medium. S-SFM, SW480 serum free medium; S-CM, SW480 conditioned medium; S-NC, SW480 negative control conditioned medium; S-ATAD2-shRNA, SW480 ATAD2-shRNA conditioned medium. Data were present as mean \pm SD. $* P<0.05 ; * * P<0.01$; $* * * \mathrm{P}<0.001$. 

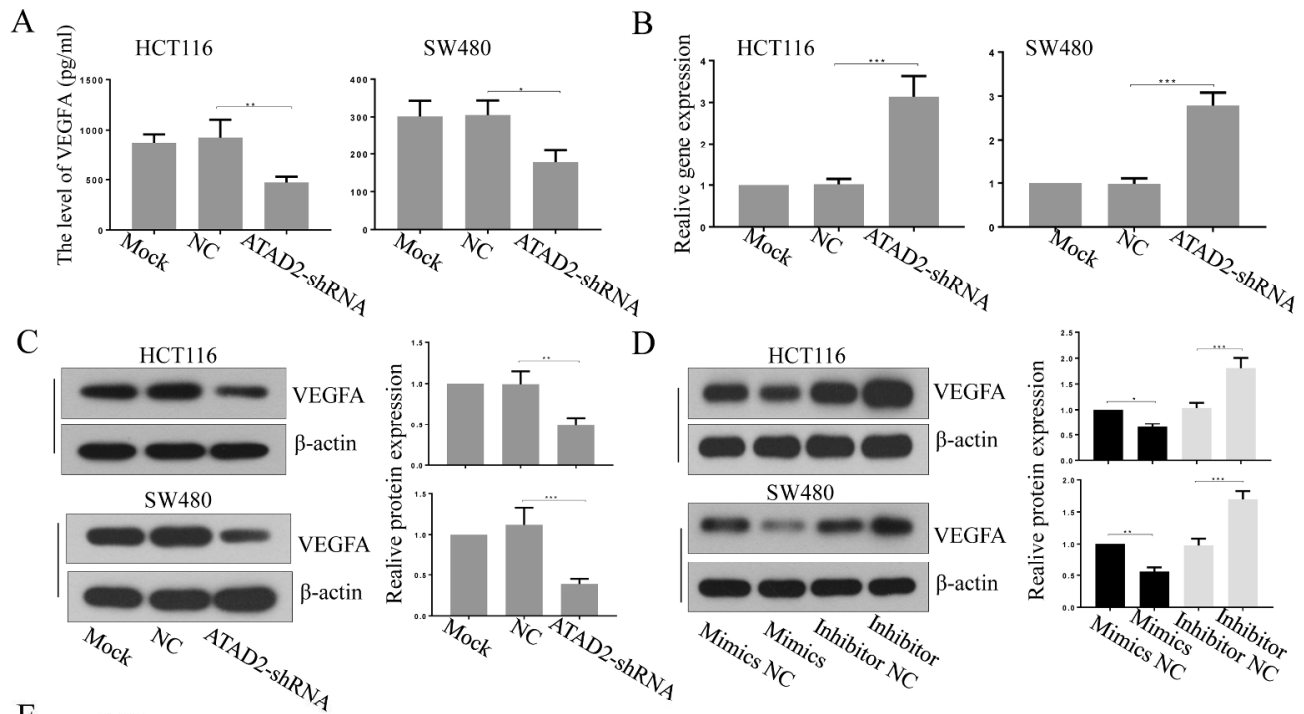

$\mathrm{E}$

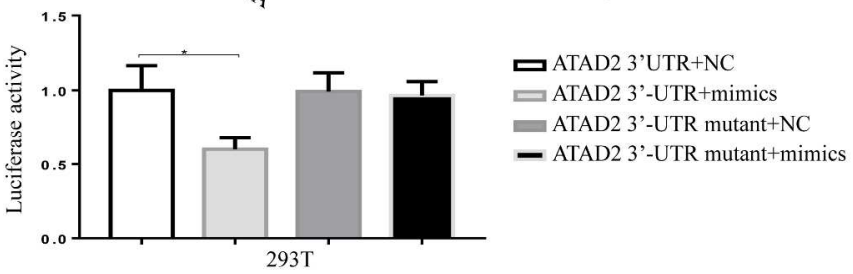

Figure 4. ATAD2 regulated the level of VEGFA via miR-520a colorectal cancer cells. Levels of VEGFA in the supernatant of ATAD2-silenced HCT116 or SW480 cells were determined by ELISA kit (A). Real-time PCR was employed to detect miR-520a expression in colorectal cancer cells with or without ATAD2 knockdown (B). The expression of VEGFA in HCT116 or SW480 cells following silencing of ATAD2 was assessed through western blotting (C). The effect of miR-520a mimics or inhibitor on the expression of VEGFA in HCT116 or SW480 cells was evaluated by western blotting (D). The effect of miR-520a mimics on luciferase activity of ATAD2 $3^{\prime}-$ UTR was determined (E). Data were present as mean $\pm \mathrm{SD}$. ${ }^{*} \mathrm{P}<0.05 ; * * \mathrm{P}<0.01 ; * * * \mathrm{P}<0.001$.

$243 \times 181 \mathrm{~mm}(300 \times 300 \mathrm{DPI})$ 
A

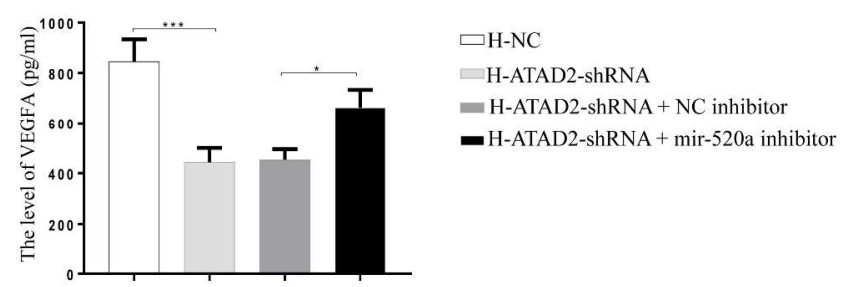

B
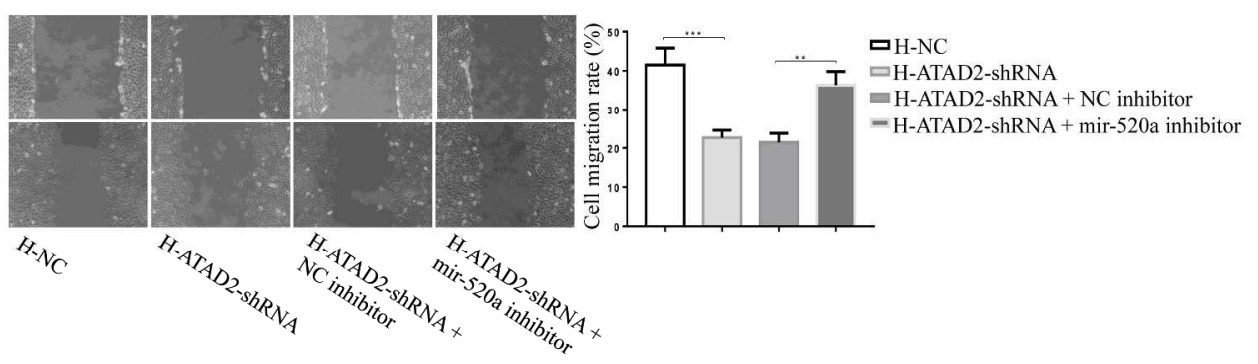

$\mathrm{C}$
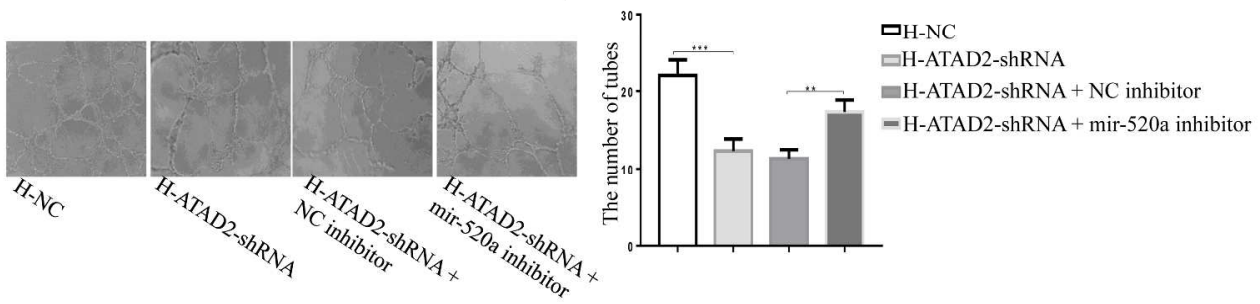

Figure 5. MiR-520a inhibitor reversed the inhibiting effect of CM from ATAD2-silenced HCT116 cells on HUVEC. Levels of VEGFA in the supernatant of ATAD2-silenced HCT116 were determined by ELISA kit (A). Migration and capillary tube formation of HUVEC were assessed by wound healing (B) and tube formation assays (C), respectively. H-NC, HCT116 negative control conditioned medium; H-ATAD2-shRNA, HCT116 ATAD2-shRNA conditioned medium. H-ATAD2-shRNA + NC inhibitor, HCT116 ATAD2-shRNA conditioned medium + . negative control inhibitor. H-ATAD2-shRNA + mir-520a inhibitor, HCT116 ATAD2-shRNA conditioned medium + mir-520a inhibitor. Data were present as mean $\pm \mathrm{SD}$. $* \mathrm{P}<0.05 ; * * \mathrm{P}<0.01$; $* * * \mathrm{p}<0.001$.

$250 \times 186 \mathrm{~mm}(300 \times 300 \mathrm{DPI})$ 
A

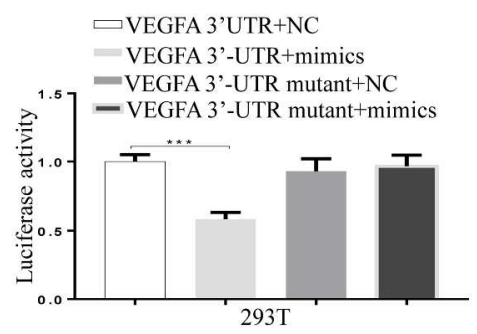

$\mathrm{C}$

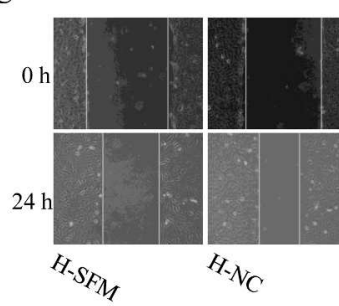

$\mathrm{D}$

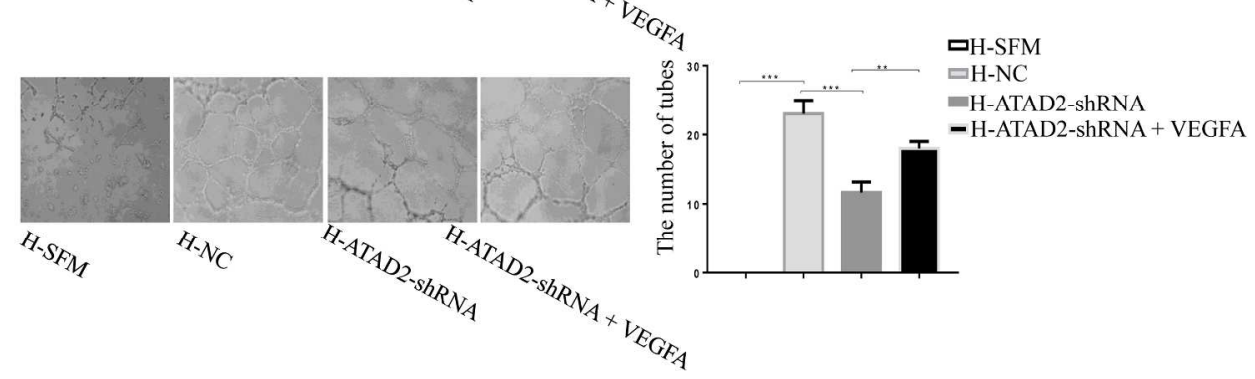

B

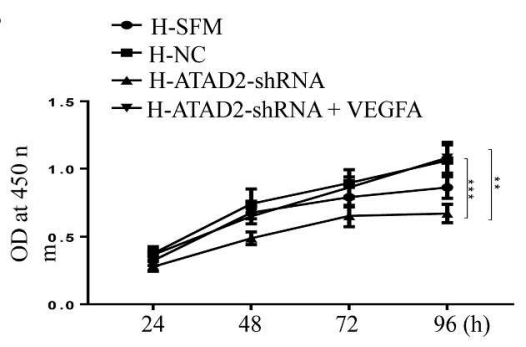

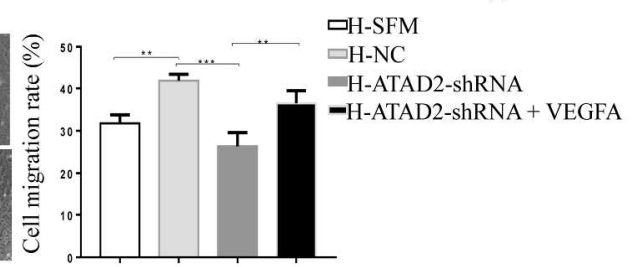

Figure 6. Treatment with VEGFA reversed the inhibiting effect of CM from ATAD2-silenced HCT116 cells on HUVEC. The effect of miR-520a mimics on luciferase activity of VEGFA 3'-UTR was assessed (A). Proliferation, migration, and capillary tube formation of HUVEC was determined by CCK-8 (B), wound healing (C), and tube formation assays (D), respectively. H-SFM, HCT116 serum free medium; H-NC, HCT116 negative control conditioned medium; H-ATAD2-shRNA, HCT116 ATAD2-shRNA conditioned medium. H-ATAD2-shRNA + VEGFA, HCT116 ATAD2-shRNA conditioned medium + $20 \mathrm{ng} / \mathrm{ml}$ VEGFA. Data were present as mean $\pm \mathrm{SD}$. $* * \mathrm{P}<0.01 ; * * * \mathrm{P}<0.001$.

$$
233 \times 190 \mathrm{~mm}(300 \times 300 \mathrm{DPI})
$$

